\title{
Beantragung der Bewilligung von akademischen Multicenterstudien bei deutschen Ethikkommissionen
}

\author{
Nicola Gökbuget $^{\mathrm{a}} \quad$ Ralph Naumann $^{\mathrm{b}}$ für den Arbeitskreis AMG der DGHO \\ a Universitäres Centrum für Tumorerkrankungen, Medizinische Klinik II, Hämatologie/Onkologie, Klinikum der Goethe-Universität, \\ Frankfurt a.M., \\ ${ }^{\mathrm{b}}$ Stiftungsklinikum Mittelrhein, Koblenz, Deutschland
}

\section{Schlüsselwörter}

Klinische Studien · Ethikkommission · Multicenterstudien

\section{Zusammenfassung}

Für klinische Studien muss vor Beginn ein positives Votum der zuständigen Ethikkommissionen eingeholt werden. In Deutschland gibt es fast 50 unabhängige Ethikkommissionen, die für die einzelnen Standorte in Multicenterstudien zuständig sein können. Da gerade bei Studien an seltenen Erkrankungen eine Beteiligung von vielen Zentren notwendig ist, stellt das Beantragungsverfahren bei akademischen Multicenterstudien für maligne hämatologische Erkrankungen häufig einen bürokratischen Albtraum dar. Einzelne Aspekte der Antragstellung sowie Ansätze für mögliche Verbesserungen werden dargestellt.

\section{Einleitung}

Multizentrische Therapieoptimierungsstudien (TOS) bei malignen hämatologischen Erkrankungen haben in Deutschland eine jahrzehntelange Tradition. Merkmal dieser Studien ist die Verbindung von Forschung und Versorgung. Gerade bei seltenen Erkrankungen kann nur in dieser Konstellation ein Erkenntnisgewinn erzielt werden. TOS zielen nicht auf die Prüfung von Medikamenten, sondern auf die Entwicklung von multimodalen Therapiestrategien, die beginnend mit einer umfassenden diagnostischen Charakterisierung über eine risikoadaptierte Therapiestratifikation den therapeutischen Einsatz von Zytostatika, Antikörpern, Wachstumsfaktoren, Strahlentherapie, Zelltherapien (z.B. Stammzelltransplantation) und Empfehlungen zur Supportivtherapie umfas-
Keywords

Clinical trials · Ethical review board · Multicenter trials

\section{Summary}

Application for Academic Multicenter Studies at German Ethical Review Boards

Before the start of a clinical trial, approval by the responsible institutional ethical review board (IRB) is required. In Germany, nearly 50 independent IRBs may be responsible for the different participating sites of a multicenter study. In trials for rare diseases, the participation of many centers is required. Therefore, the application procedure for academic multicenter trials in malignant hematologic diseases is often a bureaucratic nightmare. Different aspects of IRB application and possible approaches for improvement are presented.

sen können. Ein Prüfmedikament im eigentlichen Sinn kann in diesen Studien nicht definiert werden.

Die multizentrischen TOS wurden von deutschen Förderinstitutionen gezielt unterstützt und gehören $\mathrm{zu}$ den erfolgreichsten Modellen für die klinische Forschung [1]. Auch für die Patientenversorgung in Deutschland haben die Studien eine große Bedeutung. Institutionen der Gesundheitsversorgung wie z.B. das Institut für Qualität und Wirtschaftlichkeit im Gesundheitswesen (IQWiG), der Gemeinsame Bundesausschuss (G-BA) oder die Medizinischen Dienste der Krankenversicherung fordern in ihren Stellungnahmen immer wieder die Durchführung von unabhängigen, versorgungsnahen Studien, um unter qualitätskontrollierten Bedingungen neue oder nicht ausreichend geprüfte Therapien im Versorgungsalltag zu untersuchen [2,3].

\section{KARGER \\ Fax +497614520714 \\ Information@Karger.com}

www.karger.com (c) 2013 S. Karger GmbH, Freiburg

0378-584X/13/3614-0029\$38.00/0

Accessible online at:

www.karger.com/onk 
Schließlich sind TOS auch aus Sicht der Arzneimittelsicherheit sehr wichtig. Nachdem die Zulassung neuer Substanzen häufig auf relativ geringen Patientenzahlen und einer selektionierten Menge an Patienten basiert, müssen neue Substanzen nach der Zulassung in versorgungsnahen Therapieoptimierungen weiter beobachtet werden [4]. Wichtig ist dabei, dass gerade bei seltenen Erkrankungen der Einsatz neuer Medikamente nur selten exakt im Rahmen der Zulassung erfolgen kann. Dies ist darauf zurückzuführen, dass die Zulassungen bei seltenen Erkrankungen häufig nicht beantragt werden. Deshalb werden in TOS zwar zugelassene Medikamente eingesetzt, aber unter Bedingungen, die nicht der Zulassung entsprechen.

Bei einigen Erkrankungen stellte die Behandlung in TOS bis vor wenigen Jahren den medizinischen Standard dar und weit mehr als $50 \%$ der Patienten mit Neudiagnose wurden in Studien eingebracht. Damit wurden bei einigen Leukämien und Lymphomen schon vor vielen Jahren Studienquoten erreicht, wie sie heute als Benchmark für Comprehensive Cancer Centers mit Förderung durch die Deutsche Krebshilfe [5] oder für die Zertifizierung onkologischer Zentren gelten.

Für die Patientensicherheit haben diese Studien ebenfalls eine erhebliche Bedeutung, weil Patienten gerade mit seltenen Erkrankungen wie den hämatologischen Neoplasien die Gewissheit haben, nach umfassender Aufklärung gemäß Standards behandelt zu werden, die von Experten definiert sind, und dass die Therapie einer externen Qualitätskontrolle unterliegt. Nicht zuletzt haben die TOS bei Leukämien und Lymphomen über Jahrzehnte zu einer dramatischen Verbesserung des Langzeitüberlebens bei vielen Erkrankungen geführt.

\section{Konsequenzen der European Clinical Trials Directive und der 12. Arzneimittelgesetz-Novelle}

Im Jahr 2001 wurde die Clinical Trials-Direktive 2001/20/EC von der Europäischen Union (EU) in Kraft gesetzt und im Jahr 2004 im Rahmen der 12. Arzneimittelgesetz(AMG)Novelle und der sogenannten Good Clinical Practice(GCP)Verordnung in deutsches Recht übernommen. Diese gesetzliche Änderung hatte erhebliche negative Konsequenzen für die unabhängige akademische Forschung in Deutschland. Die Ursache liegt vor allem darin, dass für TOS und akademische Studien mit zugelassenen Substanzen ab diesem Zeitpunkt die gleichen regulatorischen Prozesse wie für Phase-I-Studien oder Zulassungsstudien der pharmazeutischen Industrie eingehalten werden mussten. Ethikkommissionen (EK), Zulassungsbehörden und lokale Überwachungsbehörden in Deutschland haben in der praktischen Umsetzung auch für diese Studien eine maximal strenge Auslegung der regulatorischen Rahmenbedingungen erkennen lassen. Zwischenzeitlich wurde vom Bundesamt für Arzneimittel und Medizinprodukte ein Dokument zu den Rahmenbedingungen für nicht- kommerzielle klinische Prüfungen herausgegeben, das aber nur eine Klarstellung, keine Erleichterung beinhaltet [6]. Eine Stellungnahme des Deutschen Bundestages hat zwar die Bedeutung der akademischen Studien hervorgehoben, aber ebenfalls keine Erleichterung der regulatorischen Rahmenbedingungen bewirkt [7].

In zahlreichen Publikationen wurde seither massive Kritik sowohl an der nationalen als auch an der europäischen Gesetzgebung geäußert [8-18]. Die EU-Kommission hat im Jahr 2011 einen öffentlichen Konsultationsprozess zur Novellierung der Direktive begonnen und in ihrer Einleitung erkennen lassen, dass die Hemmung der unabhängigen klinischen Forschung als Problem durchaus erkannt wurde [19]. Es gab eine umfassende öffentliche Reaktion auf den Konsultationsprozess $[19,20]$ und darüber hinaus eine Reihe von Initiativen, deren Ziel die Erarbeitung von Vorschlägen für die Gesetzesnovellierung war [21, 22]. Da die Novellierung der europäischen Gesetzgebung sehr langwierig ist, gefolgt von einer ebenso langwierigen Umsetzung in nationales Recht, kann eine Verbesserung der Rahmenbedingungen von akademischen klinischen Studien auf EU-Ebene nicht abgewartet werden.

\section{Beantragung von klinischen Studien bei EK}

Hauptproblem in der praktischen Aktivierung von Multicenterstudien in Deutschland ist aktuell die Beantragung bei EK. Hierbei bewertet eine federführende EK am Standort des Studienleiters die Gesamtstudie. Gleichzeitig muss die Studie bei den zuständigen EK aller beteiligten Prüfzentren eingereicht werden, deren Aufgabe es ist, die Eignung der Prüfzentren und die Qualifikation der Prüfer zu bewerten. Die beteiligten EK sollen ihre Stellungnahme der federführenden EK überleiten, die dann letztlich ein Gesamtvotum erstellt. Für das Bewertungsverfahren sind klare zeitliche Vorgaben gemacht (30 Tage), und dies ist eine der wenigen positiven Konsequenzen der 12. AMG-Novelle.

Bei einer großen Multicenterstudie kann es notwendig werden, die Anträge bei bis zu $50 \mathrm{EK}$ parallel einzureichen. Dieser Prozess wiederholt sich bei sogenannten substanziellen Amendements. Im Folgenden sollen einige praktische Erfahrungen aus der Beantragung von Multicenterstudien berichtet werden.

\section{Unterschiedliche Vorgaben}

Die Vorgaben der EK im Hinblick auf die einzureichenden Unterlagen sind nicht einheitlich. Einige Abweichungen in den Anforderungen werden erst im Laufe des Antragsverfahrens klar, weil sie auf den Webseiten der EK nicht dargestellt sind. Diese fehlende Harmonisierung resultiert letztlich aus der Unabhängigkeit der einzelnen EK, dem föderalen System in Deutschland und der Vielzahl der Träger von EK, die z.B. Universitäten oder Ärztekammern zugeordnet sind. In Teil- 
bereichen konnte eine Harmonisierung durch den Arbeitskreis der Medizinischen Ethik-Kommissionen erreicht werden, dessen Empfehlungen jedoch nicht bindend sind.

\section{Bürokratischer Aufwand}

Die Antragsunterlagen, selbst für die beteiligten EK, umfassen jeweils einen DIN-A4-Ordner. Sämtliche Unterlagen müssen üblicherweise sowohl als Papierversion als auch als elektronische Version auf CD-ROM eingereicht werden. Die Zahl der geforderten Kopien des Ordners liegt typischerweise bei 10 für die federführende EK und rangiert zwischen 2 und 9 für die beteiligten EK. Multipliziert man dies mit der Zahl der EK, kann leicht eine Zahl von 100 oder mehr Ordnern erreicht werden. Die Zahl der benötigen Kopien kann über 200000 liegen. Der erste Schritt bei der Planung einer Studieneinreichung ist somit eine Großbestellung von Büromaterial (Papier, Ordner, Trennstreifen, Kartons), die Reservierung entsprechender Räumlichkeiten zur Lagerung des Materials und Vorbereitung der Ordner und die Bereitstellung von Personal für die tagelangen Kopierarbeiten.

\section{Gebühren}

Die Gebühren für die Einreichung von AMG-Studien bei EK sind uneinheitlich. Zunächst ist - auch nach Recherche auf den Webseiten - nicht klar, ob und welche Ermäßigungen für akademisch initiierte Studien genehmigt werden. Somit kann ein Budget für diesen Teil der Studieneinreichung nicht geplant werden. Während einige EK, vorwiegend universitäre, erfreulicherweise zum Teil erhebliche Reduzierungen für akademische Studien gewähren oder vollständig verzichten, lehnen andere dies ab. Die Notwendigkeit der Gebührenerhebung wird damit begründet, dass alle am Genehmigungsprozess beteiligten Institutionen die Finanzierung der anfallenden Aufgaben selbst aufbringen müssten. Die Gebühren für die Leistungen der beteiligten EK variieren allerdings nach unserer Erfahrung zwischen 0 und 1500 Euro. Es ist unklar, worin die Unterschiede in der Gegenleistung bestehen, die eine solche Spannbreite der Gebühren rechtfertigen.

\section{Überschreitung der definierten Aufgabenbereiche}

Obwohl die Aufgabenverteilung zwischen federführender und beteiligter EK klar festgelegt ist, übernehmen beteiligte EK immer wieder Aufgabenbereiche, die eigentlich der federführenden EK obliegen. So nehmen beteiligte EK Stellung zum Protokoll oder fordern Unterlagen, die grundsätzlich von der federführenden EK zu prüfen sind, z.B. zur Probandenversicherung. Eine andere EK forderte die Qualifikationsdokumente sämtlicher Prüfer einer multizentrischen Studie. Allen beteiligten Institutionen obliegt die Verpflichtung zur Aufwandsreduzierung, unter anderem da es um die Verwendung öffentlicher Gelder geht. Es sollte Gewissheit darüber bestehen, dass alle EK nach Qualitätsstandards arbeiten und somit Doppel- und Parallelprüfungen nicht notwendig sind.

\section{Eignungsnachweis der Prüfzentren}

Der größte bürokratische Aufwand ergibt sich aus dem Sammeln und Bereitstellen der sogenannten Qualifikationsunterlagen. Dabei ist zu bedenken, dass die Therapie hämatologischer Neoplasien an den hämatologisch-onkologischen Abteilungen deutscher Krankenhäuser stattfindet. Solange die Kostenträger keine allgemeingültigen Qualitätsstandards für die Behandlung festlegen, wie dies z.B. in Vereinbarung des G-BA für die Kinderonkologie geschehen ist [23], wird die Behandlung auch weiterhin in der Breite der genannten Spezialabteilungen stattfinden. Eine über diese, für die allgemeine Versorgung der Patienten geltende Qualifikation von Zentren kann für TOS nicht definiert werden, die per definitionem ja möglichst versorgungsnah durchgeführt werden sollen. Im Gegenteil ist eine möglichst breite Beteiligung sinnvoll, um für viele Patienten eine qualitätskontrollierte Studientherapie zu ermöglichen. Somit würde es genügen, die Qualifikation dieser Zentren für die Durchführung von TOS einmalig festzustellen, zumal die jeweiligen Abteilungen den zuständigen EK gut bekannt sind.

Die Realität sieht allerdings anders aus. Bei Einbeziehung von Spezialpraxen könnten 300 oder mehr Studienzentren für die Beteiligung an einer TOS infrage kommen. Für jede einzelne Studie muss erneut ein entsprechendes Formular zur Eignung des Zentrums ausgefüllt werden. Hier muss unter anderem selbst für Universitätskliniken bestätigt werden, dass eine Notfallversorgung besteht. Fehlt nur eine Angabe, werden Nachfragen gestellt. Weiterhin wird die Zahl der erwarteten Patienten dokumentiert und die Zahl der gegebenenfalls parallel laufenden Studien. Einzelne EK fordern Listen sämtlicher am Zentrum durchgeführten Studien. Begründet wird dies mit der Notwendigkeit, dass die EK prüfen müsse, ob eventuell zu viele parallele Studien durchgeführt werden, die vom verfügbaren Team nicht bewältigt werden können. Eine solche Prüfung ist aber wegen des für die verschiedenen Studien sehr unterschiedlichen Aufwands gar nicht möglich. Die Verantwortung für die Arbeitsorganisation sollte stattdessen dem Abteilungsdirektor überlassen werden.

\section{Qualifikationsnachweis der Prüfer}

Ein noch größerer Aufwand ist mit dem Qualifikationsnachweis der Prüfer verbunden. Hierbei geht es darum, formal zu prüfen, ob die Prüfärzte durch Aus- und Weiterbildung sowie berufliche Erfahrung qualifiziert sind, ausreichend Zeit für die klinische Prüfung haben, mit dem Prüfplan und Prüfpräparat vertraut sind und die gesetzlichen Regularien kennen. In $\S 40$ Abs. 1 AMG ist festgelegt, dass die Anforderungen der GCP gemäß Artikel 1 Abs. 3 der Note for Guidance on Good Clinical Practice (CPMP/ICH/135/95) einzuhalten sind. Die Bundesärztekammer hat eine Empfehlung für EK entwickelt, die in ihren Details auf diese Guidance (Anleitung) Bezug nimmt [24]. Hier wird der volle Umfang des sogenannten Qualifikationsnachweises unabhängig von der Art der Studie gefordert. 
Es war bisher allgemeiner Konsens, dass alle Ärzte, die studienspezifische Aufgaben wahrnehmen, als Prüfer gemeldet werden müssen. Dazu gehören unter anderem die Aufklärung der Patienten, die Prüfung der Einschlusskriterien, die Bewertung von unerwünschten Ereignissen und die Unterzeichnung von Dokumentationsunterlagen. In TOS, die in der Standardversorgung durchgeführt werden, konnte es notwendig werden, 2-3 Prüfer für jede beteiligte Station oder Ambulanz zu melden. Nach den üblichen halbjährlichen Rotationen, die im Rahmen der Facharztausbildung anfallen, wurden entsprechend umfangreiche Nachmeldungen notwendig. Häufig war das Team nach Abwicklung der langwierigen Antragstellung schon zum Studienstart nicht mehr aktuell und es mussten unmittelbar Nachmeldungen erfolgen, die dann wieder zu Kosten und Verzögerungen des Studienstarts führen. Die Zusammenstellung der Unterlagen ist überhaupt nur möglich, wenn in der beteiligten Klinik eine Study Nurse mehrere Stunden Arbeit investiert, um die Dokumente zu kopieren, gegebenenfalls vorauszufüllen und dann auf den Stationen und Ambulanzen die entsprechenden Unterschriften einzuholen. Es bleibt offen, wie solche Aufgaben von den Kliniken finanziert werden sollen.

Obwohl die Ärzte oft für verschiedenste Studien als Prüfer gemeldet sind und die Dokumente der EK bereits vorliegen, müssen die Qualifikationsunterlagen bei jeder Studie erneut eingereicht werden. Dazu gehört im Allgemeinen ein berufsbezogener Lebenslauf, der aktuell unterschrieben werden muss. Der Begriff «aktuell» wird unterschiedlich definiert. Es gibt Beispiele, dass EK einen Lebenslauf, der vor mehr als einem halben Jahr unterschrieben wurde, nicht mehr als aktuell anerkennen. So kann die Situation eintreten, dass während des langwierigen Zusammentragens von Unterlagen in einer Multicenterstudie die ersten eingegangenen Unterlagen schon wieder veraltet sind, wenn die Dokumente des letzten Zentrums gerade komplettiert werden. Immer wieder wird in diesem Zusammenhang auch diskutiert, ob ein Lebenslauf eine Originalunterschrift tragen muss oder ob Kopien ausreichend sind. Durch die strikte Auslegung des Begriffs «aktueller Lebenslauf» wird verhindert, dass z.B. für den Zeitraum eines Jahres vorhandene, unterzeichnete Lebensläufe von Prüfern für die anstehenden Einreichungen kopiert werden.

Als Bestandteil der Qualifikationsunterlagen wird auch der Nachweis einer Schulung in GCP gesehen. Als Grundlage wird dabei die Vorgabe der oben genannten Guidance zitiert, die besagt, dass Ärzte entsprechende Kenntnisse aufweisen müssen, wobei nicht definiert ist, wie sie sich die Kenntnisse aneignen können. Es wäre durchaus denkbar, dass ein Arzt bestätigt, sich die Kenntnisse durch Selbststudium angeeignet zu haben. In der Praxis werden jedoch Teilnahmebestätigungen von GCP-Kursen gefordert. Hierbei wird unterschiedlich ausgelegt, wie umfangreich diese Kurse sein müssen. Teilweise wird zwischen erfahrenen und weniger erfahrenen Prüfern unterschieden, die dann Kurse von weniger oder mehr als einem Tag Dauer nachweisen sollen. Obwohl diese differenzierte Vorgehensweise im Einzelfall hilfreich sein kann, ist es leicht vorstellbar, wie schwer diese unterschiedlichen Vorgaben bei der Zusammenstellung der Unterlagen für Multicenterstudien berücksichtigt werden können. Hier ist inzwischen ein neues Geschäftsmodell der Kursanbieter entstanden, die durchaus daran interessiert sind, die Anforderungen zu verschärfen.

Ein zusätzlicher Diskussionspunkt ist die Notwendigkeit von Updates zu den GCP-Kenntnissen. Hier gibt es keinerlei gesetzliche Vorgabe und dennoch eine große Bandbreite von Ansichten bei den EK, die von einem jährlichem Update zu verschiedenen Themen bis zur Wiederholung eines vollständigen GCP-Kurses alle 2 Jahre reicht. Andere EK fordern nach jeder AMG-Novelle ein Update, selbst wenn keine für akademische Studien relevanten Inhalte geändert wurden. Man könnte durchaus die Prozesse erleichtern, indem man annimmt, dass die regelmäßige aktive Teilnahme an klinischen Studien ein ausreichendes Update darstellt, zumal bei den Studien im Rahmen der Initiierung heutzutage immer auch GCP-Themen angesprochen werden.

Als weiterer Aspekt der Qualifikation wird die Studienerfahrung gesehen. Deshalb fordern die EK im Lebenslauf eine umfangreiche Auflistung aller Studien, an denen der Prüfarzt bisher teilgenommen hat und aktuell teilnimmt, wobei Beginn und Ende, Phase, Titel und die genaue Funktion gefordert werden. Wenn neue Studien der EK bereits aus Antragstellungen bekannt sind, der Lebenslauf aber schon z.B. ein halbes Jahr vorher erstellt wurde, kann dies wieder zu Nachforderungen führen.

Einige EK betreiben eine noch weitergehende berufliche Qualifikationsprüfung. Bei Prüfern, deren Vorgesetzter der Übertragung der Funktion zugestimmt hat, wird trotzdem die Qualifikation infrage gestellt. So werden Schulungen für bestimmte Erkrankungen gefordert oder es wird festgestellt, dass Assistenzärzte mit weniger als 1 Jahr Fortbildungszeit grundsätzlich nicht als Prüfärzte geeignet sind oder aber nur eine bestimmte Zahl von Studien bewältigen können.

Schließlich muss jeder Prüfarzt ein Formular zum sogenannten Financial Disclosure ausfüllen. Damit soll sichergestellt werden, dass er im Rahmen der Studie keine eigenen finanziellen Interessen verfolgt. Selbst in Studien der pharmazeutischen Industrie ist schwer vorstellbar, wie ein einzelner Prüfarzt, der z.B. über ein Aktienpaket einer Pharmafirma verfügen könnte, durch seine begrenzten Studienaktivitäten einen Einfluss auf die finanzielle Situation der Firma nehmen sollte. In akademisch initiierten Studien ist das Formular noch weniger sinnvoll, wird aber dennoch routinemäßig gefordert.

Einzelne EK gehen über diesen Satz von Dokumenten noch hinaus und fordern beispielsweise Kopien der Approbationsurkunden. Zur Begründung wird angeführt, dass Prüfer Ärzte sein müssen und dies von der EK geprüft werden müsse. Diese Tatsache müsste allerdings bei der Einstellung der Person in der entsprechenden Klinik übersehen worden sein, und auch der Abteilungsdirektor, der ja das Prüferteam bestätigt, dürfte dies nicht bemerkt haben. 
Weitere Diskussionen ergeben sich bei der Frage, ob auch Qualifikationsdokumente für Studienassistenzpersonal gesammelt werden müssen. Einzelne EK bestehen darauf, Lebensläufe und GCP-Fortbildungen auch dieser Personen in Kopie zu erhalten. Dahinter steckt möglicherweise der Verdacht, dass Prüfer Personal einsetzen, das nicht ausreichend qualifiziert ist.

Insgesamt dauert es regelhaft viele Monate, bis sämtliche Dokumente von Prüfärzten aus 100 oder mehr Kliniken für die Ersteinreichung einer Studie eingesammelt, sortiert und überprüft sind. Dieser Prozess wird dann während der gesamten Studienlaufzeit fortgesetzt, da sich die zuständigen Prüfer immer wieder ändern.

\section{Studien bei seltenen Erkrankungen}

Im aktuellen System sind Studien bei seltenen Erkrankungen praktisch nicht mehr durchführbar. In solchen Studien müssten sämtliche oben genannten Prozesse für eine Vielzahl von Zentren durchgeführt werden, die möglicherweise während der ganzen Laufzeit der Studie keinen Patienten einbringen können. Weder die beteiligten Kliniken noch Studienzentralen können diesen Aufwand leisten. Für die Versorgung von Patienten mit seltenen Erkrankungen, die gesundheitspolitisch immer wieder als wichtiges Ziel benannt wird, hat das gravierende Konsequenzen. Dies gilt umso mehr, als aufgrund der Diversifizierung der Therapien (z.B. zielgerichtete Substanzen für molekulare Subgruppen der Leukämien) der Begriff der «seltenen Erkrankungen» immer weiter auszulegen ist. Dazu gehören z.B. sämtliche akuten Leukämien, Subgruppen wie die Philadelphia-Chromosom( $\mathrm{Ph}$ )-positive akute lymphatische Leukämie (ALL), Rezidive hämatologischer Erkrankungen oder auch Patientengruppen wie ältere Patienten.

\section{Register und epidemiologische Projekte}

Für epidemiologische Studien, die nicht dem AMG unterliegen, gelten grundsätzlich andere Vorgaben. Die Studien werden bei der EK des Projektleiters eingereicht. Dann besteht für die beteiligten Ärzte nach Berufsrecht die Pflicht einer Beratung bei der jeweils zuständigen EK. Um zum Ablauf genauere Informationen zu erhalten, muss man in die Berufsordnungen der einzelnen Bundesländer Einsicht nehmen. Hier steht in einigen Fällen, dass für das jeweilige Bundesland eine einzige Beratung ausreicht. In anderen Fällen sind für jeden Standort erneute Beratungen notwendig. Es kommt auch vor, dass entgegen der eigenen Berufsordnung Beratungen gefordert werden. Für die Beratung müssen bei multizentrischen Projekten erneut umfangreiche Unterlagen zusammengestellt werden. Jede EK hat eigene Formulare und eigene Vorgaben zur Zahl der Kopien. Bei vielen Varianten der Formulare fällt auf, dass diese auf AMG-Studien ausgelegt sind und für epidemiologische Projekte nur schwerlich sinnvoll ausgefüllt werden können. Auch werden in Einzelfällen auch Lebensläufe von teilnehmenden Ärzten gefordert, obwohl die Prüfung der Qualifikation bei epidemiologischen
Studien nicht zu den Aufgaben der EK gehört. Schließlich fallen wieder Gebühren zwischen 0 und 1500 Euro an. Da es nur um eine berufsrechtliche Beratung geht, stellt sich die Frage, warum die EK nicht selbst versuchen, den Aufwand zu begrenzen, und sich einfach dem Votum einer anderen EK anschließen. Auch hier wird wieder deutlich, dass die EK nur zum Teil bereit sind, die Qualität der Arbeit anderer EK anzuerkennen, und Wert darauf legen, eigene Auslegungen bestimmter Aspekte in die Beratung einzubringen.

\section{Novellierung des AMG und der GCP-Verordnung}

Im Oktober 2012 wurde in Deutschland ein zweites Gesetz zur Änderung arzneimittelrechtlicher und anderer Vorschriften beschlossen. Eine wesentliche Erleichterung der Ethikbeantragung von TOS könnte sich theoretisch aus der Änderung von $\S 40$ (1a) ergeben. In der Neufassung wird vorgeschlagen, dass ein Prüfer als Leiter einer Prüfgruppe am Zentrum die Verantwortung für die Durchführung der Studie trägt. Auch wenn der Gesetzgeber in den Erläuterungen verdeutlicht, dass eine Erleichterung der Beantragungsverfahren angestrebt wird, lässt die Formulierung erneut Spielraum für breit gefasste Interpretationen unterschiedlicher EK. Erste Erfahrungen mit der Umsetzung der gesetzlichen Änderung zeigen eine große Spannbreite der Auslegungen und ebenso große Verwirrung im Hinblick auf die Vorgaben und die Gültigkeit der Änderungen bei bereits laufenden Studien. Es gibt wiederum keine Verpflichtung der EK zu einheitlichen Vorgehensweisen. Beunruhigend ist, dass auch die Regierungsbehörden keine einheitliche Auslegung finden. Studienleitungen und Prüfzentren können zum Teil nicht mehr einschätzen, nach welchen gesetzlichen Vorgaben sie in den einzelnen Studien arbeiten müssen.

Aus der Sicht akademischer Studiengruppen wäre es sinnvoll, die Überwachung der Prüfgruppe auf den Prüfer und den Stellvertreter zu beschränken. Der Prüfer sollte dann in der Übernahme seiner Verantwortung den üblichen Überwachungsmechanismen z.B. durch lokale oder zentrale Behörden unterliegen. Dies kann keine Aufgabe von EK sein. Aufgrund der oben geschilderten Erfahrungen besteht große Skepsis, ob die Änderung des AMG in der Praxis zu einer tatsächlichen und zügigen Erleichterung der Antragsverfahren führen wird.

\section{Fazit}

Grundsätzlich ist festzustellen, dass die Mehrzahl der EK kooperativ arbeitet und Forschungsaktivitäten durch eine professionelle inhaltliche Beratung unterstützt. Das Ziel der EK, Patientenrechte zu schützen, kann nur bejaht werden. Dennoch ist leider die EK-Beantragung von multizentrischen TOS zu einem bürokratischen Großprojekt geworden - obwohl gerade diese Studien auch dem Patientenschutz dienen. 
Multicenterstudiengruppen haben in den vergangenen Jahren zwar die Kompetenz zur Erfüllung der Vorgaben erworben. Dennoch hat sich der Aufwand für jedes einzelne Projekt erheblich erhöht und entsprechend sind die Kosten gestiegen. In der Konsequenz können weniger Studien durchgeführt werden, Studien mit einer beschränkten Zahl von Zentren werden favorisiert und es müssen hohe Beträge für die Finanzierung aufgebracht werden. Da die Fördermittel aus öffentlichen Geldern begrenzt sind, geht der Trend zu Studien, die eine Kofinanzierung durch die pharmazeutische Industrie haben.

Auch für die beteiligten Kliniken ist der Aufwand der Studienteilnahme kaum noch zu leisten, zumal in TOS keine oder nur sehr geringe Fallgelder gezahlt werden können. Es gibt keine Gelder, weder von Kostenträgern noch von Klinikleitungen, die für die Finanzierung solcher Aufgaben zur Verfügung stehen.

Auch Patienten leiden unter den komplexen Prozessen. So werden weniger Zentren für Studien aktiviert und Patienten müssen zum Teil weite Reisen auf sich nehmen. Für viele, gerade seltene Erkrankungen können keine TOS mehr angeboten werden. Zusätzlich wird mit dem Ziel der Absicherung von Sponsoren die schriftliche Patientenaufklärung immer umfangreicher und ist für einen Patienten in einer emotionalen Stresssituation kaum noch zu erfassen.

Insgesamt ist es sehr bedauerlich, dass die Prüfungen der EK fast ausschließlich formaler Natur sind. Anfragen bei Leitungen akademischer Studien bestätigen, dass inhaltliche Fragen der Studien kaum noch diskutiert werden. Dazu kommt, dass Antragstellern in strittigen Fällen ganz klar vermittelt wird, dass nur bei Erfüllung sämtlicher formaler Anforderungen der jeweiligen EK ein positives Votum erstellt wird. Viele der Anforderungen beruhen zwar nur auf Auslegungen. Selbst wenn solche Auslegungen im Widerspruch zu einer Vielzahl anderer EK stehen, gibt es aber keine Möglichkeit, einen Widerspruch durchzusetzen oder an unabhängiger Stelle Beschwerde einzulegen.

Letztlich wird die Frage, ob die umfassende Dokumentation bei der EK-Beantragung sowie andere Prozesse der Studienaktivierung, Durchführung und Überwachung tatsächlich der Patientensicherheit dienen, nicht mehr gestellt. Die ICH-GCP (ICH = International Conference on Harmonisation) wird als Standard für klinische Studien aller Art herangezogen. Demgegenüber wird in ICH-E6 die Leitlinie für Studien empfohlen, die bei Überwachungsbehörden eingereicht werden [25]. Zu keinem Zeitpunkt wurde überprüft, ob die ICH-GCP tatsächlich zu einer Verbesserung der Patienten- und Datensicherheit beiträgt. Dennoch bildet das Regelwerk jetzt die Grundlage für gesetzliche Vorgaben für alle Studienformen, ohne hinterfragt zu werden. Es bleibt unklar, warum so umfangreiche materielle und personelle Ressourcen für Aufgaben bereitgestellt werden müssen, die nie systematisch im Hinblick auf bestimmte Zielkriterien geprüft wurden.
Die Verantwortung hierfür trägt der Gesetzgeber. Die EK erfüllen - nach ihrer Auslegung - nur gesetzliche Vorgaben. In Deutschland wurde versäumt, die von der EU eigentlich intendierte Harmonisierung und Vereinfachung der EK-Beantragung umzusetzen. Die positive Motivation des Patientenschutzes führt im Rahmen eines komplexen, multizentrischen Systems zu einer vorwiegend an Formalaspekten orientierten Prüfung, die durch fehlende Harmonisierung und Machtfülle einzelner beteiligter EK verkompliziert wird. Hinter der strikten Interpretation von gesetzlichen Vorgaben steht zum Teil die Sorge der Beteiligten, bei Fehlentscheidungen juristisch belangt werden zu können. Die umfassende Dokumentation formaler Schritte dient der Absicherung. Für viele der aufgeführten Maßnahmen jedoch ist ein positiver Effekt auf den Patientenschutz kaum vorstellbar. Die Politik muss Rechtssicherheit schaffen und entscheiden, ob die knappen Ressourcen für Forschung und Gesundheitsversorgung in der bisherigen Weise auch weiterhin für bürokratische Prozesse eingesetzt werden sollen. In der Konsequenz bedeutet dies nämlich, dass auf der Grundlage unbewiesener Vorteile für die Patientensicherheit die unabhängige akademische Forschung geschwächt und die Patientenversorgung verschlechtert wird.

\section{Vorschläge zur Verbesserung der Rahmenbedingungen für TOS}

Es gibt mehrere Maßnahmen, die zu einer Erleichterung der Situation beitragen könnten:

- Am wichtigsten wäre die Stärkung der Prüfarztverantwortung. Der Hauptprüfer jedes Zentrums und der Abteilungsdirektor, der einer Studienteilnahme zustimmt, sollten die Gesamtverantwortung für die Studie am Zentrum übernehmen. Nur der Hauptprüfer sollte den EK gemeldet werden. Hier würde eine einmalige Bereitstellung von Qualifikationsdokumenten, z.B. mit jährlichem Update, ausreichen. Innerhalb des Zentrums muss der Hauptprüfer dann Aufgaben delegieren und die Qualifikation des Teams sicherstellen und dokumentieren. Dies kann gegebenenfalls bei Inspektionen überprüft werden und entspricht dem Geist der jetzt vorgelegten Änderung des AMG.

- Kurzfristige Aktivierung von zusätzlichen Zentren im Rahmen von Studien bei seltenen Erkrankungen, für die bereits ein Ethikvotum einer federführenden EK vorliegt. Dies muss innerhalb weniger Tage geschehen, wenn ein Patient zur Therapie vorgestellt wird. Einzelvoten bei individuellen Heilversuchen zeigen, dass dies organisatorisch durchaus möglich ist.

- Die Definition nichtinterventioneller Studien, die nicht dem AMG unterliegen, sollte auf TOS erweitert werden, da diese Studien nicht der Arzneimittelprüfung dienen. Dadurch würden zahlreiche regulatorische Prozesse und 
die Notwendigkeit einer Probandenversicherung ganz entfallen. Alternativ möglich - allerdings in der Umsetzung deutlich komplizierter und langwieriger - wäre die Entwicklung einer risikoadaptierten Studiendefinition und daran angepasster regulatorischer Vorgaben.

- Die Bereitstellung von Qualifikationsunterlagen sollte elektronisch erfolgen. Das Kompetenznetz Leukämien hat zu diesem Zweck eine internetbasierte Datenbank (www. quali-pro.de) entwickelt. In diese Datenbank können alle Qualifikationsunterlagen eingestellt werden. Die EK könnten dann per Online-Zugriff die Unterlagen einsehen und bestätigen. Der Versand von Papierdokumenten und deren Archivierung könnte so erheblich reduziert werden.

- In akademisch initiierten Studien sollte grundsätzlich auf Financial Disclosures verzichtet werden. Die EK sollten ihre Aufgabenbereiche strikt einhalten und verpflichtet werden, den Aufwand zu minimieren.

- Für akademische Studien sollten einheitliche und reduzierte Gebühren bzw. ein Gebührenverzicht angeboten werden.
- EK sollten bei unbestrittener inhaltlicher Unabhängigkeit verpflichtet werden, sich auf einheitliche Auslegungen formaler Aspekte zu einigen. Für die Klärung strittiger Anforderungen durch EK müsste eine Schiedsstelle eingerichtet werden.

Rasche Maßnahmen sind dringend erforderlich, um die Strukturen der unabhängigen akademischen Forschung in TOS vor einer dauerhaften Schädigung zu bewahren. Die Frage, wie ein Arzt Verantwortung übernimmt und trägt, lässt sich weder im Bereich klinischer Studien noch im Gesundheitssystem insgesamt in ein Regelwerk einordnen und nach Aktenlage kontrollieren. Es ist berufsimmanent, dass ein gewisses $\mathrm{Maß}$ an Vertrauen notwendig ist. Das sollte man anerkennen, anstatt die wahre Problematik zu verdecken, indem man formale Aspekte möglichst umfassend abarbeitet.

\section{Disclosure Statement}

Keine Interessenkonflikte bezüglich des Artikels.

\section{Literatur}

1 Gökbuget N, Hoelzer D: Bedeutung von Multicenterstudiengruppen für die klinische Forschung in der Hämatologie und Onkologie. Bundesgesundheitsbl 2009;52:417-424.

2 N05-03A. Stammzelltransplantation bei akuter lymphatischer Leukämie (ALL) und akuter myeloischer Leukämie (AML) bei Erwachsenen. www.iqwig.de.

3 Beschluss des Gemeinsamen Bundesausschusses über eine Änderung der Richtlinie Methoden Krankenhausbehandlung: Autologe Stammzelltransplantation bei akuter lymphatischer Leukämie (ALL) bei Erwachsenen, vom 21. Juli 2011. www.g-ba.de.

4 Schott G, Gökbuget N, Pachl H, Ludwig W-D: Klinische Studien in der Onkologie - Defizite und Lösungsvorschläge. Z Evid Fortbild Qual Gesundhwes 2011;105:657-664.

5 Deutsche Krebshilfe Program for the Development of Interdisciplinary Oncology Centers of Excel lence in Germany - Second Call for Proposals. www.krebshilfe.de.

6 Bekanntmachung des Bundesinstituts für Arzneimittel und Medizinprodukte, des Paul-Ehrlich-Instituts und des Bundesministeriums für Gesundheit vom 21. Oktober 2009. Nicht-kommerzielle klinische Prüfungen: Zusammenfassung der regulatorischen Voraussetzungen.

7 Nichtkommerzielle klinische Studien in Deutschland. Deutscher Bundestag Drucksache 17/1929, 17. Wahlperiode, 07.06.2010.

8 Elwyn G, Seagrove A, Thorne K, Cheung WY: Ethics and research governance in a multicentre study: add 150 days to your study protocol. BMJ 2005;330:847. $\checkmark$ Frewer LJ, Coles D, Champion K, et al.: Has the European Clinical Trials Directive been a success? BMJ 2010;340:c1862

10 Hackshaw A, Farrant H, Bulley S, Seckl MJ, Ledermann JA: Setting up non-commercial clinical trials takes too long in the UK: findings from a prospective study. J R Soc Med 2008;101:299-304.

11 Hearn J, Sullivan R: The impact of the 'Clinical Trials' directive on the cost and conduct of noncommercial cancer trials in the UK. Eur J Cancer 2007;43:8-13.

12 Hemminki A, Kellokumpu-Lehtinen PL: Harmful impact of EU clinical trials directive. BMJ 2006; 332:501-502.

13 Tyndall A: Why do we need noncommercial, investigator-initiated clinical trials? Nat Clin Pract Rheumatol 2008;4:354-355.

14 Robison K, Andrews PJ: '(More) trials and tribulations': the effect of the EU directive on clinical trials in intensive care and emergency medicine, five years after its implementation. J Med Ethics 2010; 36:322-325.

15 European Cancer Organisation backs proposals to revise EU Clinical Trials Directive. Expert Rev Anticancer Ther 2010;10:301-303.

16 Hoey R: The EU clinical trials directive: 3 years on. Lancet 2007;369:1777-1778.

17 Who's afraid of the European clinical trials directive? Lancet 2003;361:2167.
18 Frewer LJ, Coles D, van der Lans IA, Schroeder D, Champion K, Apperley JF: Impact of the European Clinical Trials Directive on prospective academic clinical trials associated with BMT. Bone Marrow Transplant 2011;46:443-447.

19 http://ec.europa.eu/health/human-use/consultations/ index_en.htm.

20 www.leukemia-net.org/content/international_trials/ basic information/.

21 Bilbault P, Belorgey C, Zannad F, Golinelli D, Pletan Y; participants of Round Table no 4 of Giens XXV: Clinical trials legislation - preparing for the revision of the European Directive scheduled for 2011. Therapie 2010;65:301-308.

22 Road Map Initiative for Clinical Research in Europe. www.efgcp.be/ - Events - Past Events.

23 Bekanntmachung eines Beschlusses des Gemeinsamen Bundesausschusses über die Vereinbarung zur Kinderonkologie vom 16. Mai 2006. www.g-ba.de/ informationen/beschluesse/290/.

24 Empfehlungen zur Bewertung der Qualifikation von Prüfern und Geeignetheit von Prüfstellen durch Ethik-Kommissionen bei klinischen Prüfungen nach dem AMG. Dtsch Arztebl 2010;107:A48A51.

25 Grimes DA, Hubacher D, Nanda K, Schulz KF: The Good Clinical Practice guideline: a bronze standard for clinical research. Lancet 2005;336:172-174. 